OPEN ACCESS

Edited by:

Menghong $\mathrm{Hu}$,

Shanghai Ocean University, China

Reviewed by:

Jun Jiang,

Sichuan Agricultural University, China

Brad Buckley,

Portland State University,

United States

*Correspondence:

Zhi Luo

luozhi99@mail.hzau.edu.cn; luozhi99@aliyun.com

Specialty section: This article was submitted to

Aquatic Physiology,

a section of the journal

Frontiers in Physiology

Received: 13 February 2019

Accepted: 08 July 2019

Published: 02 August 2019

Citation:

Li D-D, Ling S-C, Wu K and Luo Z (2019) Identification of Five Key

Genes Involved in Intrinsic Apoptotic Pathway From Yellow Catfish Pelteobagrus fulvidraco and Their

Transcriptional Responses to High Fat

Diet (HFD). Front. Physiol. 10:921.

doi: 10.3389/fphys.2019.00921

\section{Identification of Five Key Genes Involved in Intrinsic Apoptotic Pathway From Yellow Catfish Pelteobagrus fulvidraco and Their Transcriptional Responses to High Fat Diet (HFD)}

\author{
Dan-Dan Li ${ }^{1}$, Shi-Cheng Ling ${ }^{1}$, Kun Wu ${ }^{1}$ and Zhi Luo ${ }^{1,2 *}$ \\ ${ }^{1}$ Key Laboratory of Freshwater Animal Breeding, Ministry of Agriculture, Fishery College, Huazhong Agricultural University, \\ Wuhan, China, ${ }^{2}$ Laboratory for Marine Fisheries Science and Food Production Processes, Qingdao National Laboratory \\ for Marine Science and Technology, Qingdao, China
}

The hypothesis of the present study is that apoptosis through an intrinsic mitochondrial pathway may mediate high fat diet (HFD)-induced changes in the metabolism of Pelteobagrus fulvidraco. To this end, we cloned the full-length cDNA sequences of Cycs, Apaf1, Casp9, Casp3a, and Casp3b involved in the mitochondria apoptotic pathway, and explored their mRNA tissue expressions and transcriptional responses to HFD. All of these members shared similar domains to their orthologous vertebrate genes. They were constitutively expressed in all analyzed tissues but varied from tissue to tissue. Compared to the control, HFD up-regulated the mRNA expression of partial genes among these five key genes (Cycs, Apaf1, Casp9, Casp3a, and Casp3b) in mesenteric fat, intestine, ovary and the kidney, indicating the induction of apoptosis in these tissues; in contrast, HFD down-regulated mRNA levels of partial genes among the five key genes (Cycs, Apaf1, Casp9, Casp3a, and Casp3b) in the heart, spleen and gill tissues, indicating the inhibition of apoptosis in these tissues. The present study will facilitate further exploration into the functions of these genes at the molecular level and disclose the critical involvement of these genes against nutrient changes, indicating that processes of apoptosis in various tissues may differentially be modified by HFD.

Keywords: apoptosis, intrinsic mitochondrial pathway, gene characterization, Pelteobagrus fulvidraco, high fat diet

\section{INTRODUCTION}

Apoptosis is a highly regulated and conserved form of programmed cell death for multicellular organisms, which is trigged by a variety of physiological or pathological stimuli (Mu et al., 2010). In mammals, two main pathways triggering apoptosis, the extrinsic/death receptor pathway and the intrinsic/mitochondrial pathway, have been described, respectively (Marsden and Strasser, 2003).

Abbreviations: Apaf1, apoptotic peptidase activating factor-1; Casp3a, caspase 3a; Casp3b, caspase 3b; Casp9, caspase 9; Cycs, cytochrome c; ELFA, translation elongation factor; GAPDH, glyceraldehyde-3-phosphate dehydrogenase; HPRT, hypoxanthine-guanine phosphoribosyltransferase; RPL7, ribosomal protein L7; SEM, standard error of mean; TBP, TATAbox-binding protein; TUBA, tubulin alpha chain; UBCE, ubiquitin-conjugating enzyme. 
For the intrinsic pathway, upon induction of apoptosis, proapoptotic proteins can trigger mitochondrial outer membrane permeabilization and release of cytochrome $c$ (Cycs) into the cytosol (Liu et al., 1996). Cytochrome $c$ then binds to apoptotic protease-activating factor-1 (Apaf1) forming the Apaf1/cytochrome c complex (Jiang and Wang, 2000). The complex facilitates the activation of caspase 3 (Casp3) by caspase 9 (Casp9), finally leading to apoptotic cell death (Wang, 2001; Li et al., 2003).

Cycs, Apaf1, Casp9, and Casp3 are the key genes involved in the mitochondrial apoptotic pathway, and their identification was the first important step in characterizing the role and mechanism of apoptosis. During the last few years, the structure, regulation and the function of Cycs, Apaf1, Casp9, and Casp3 in apoptosis had been extensively investigated in mammals (Zou et al., 1997; Reis et al., 2007b; Kumaresan et al., 2016). However, information about their molecular characterization and tissue expression profiles is very limited in fish. At present, Casp9 has been characterized in sea bass (Dicentrarchus labrax) (Reis et al., 2007a), common carp (Cyprinus carpio) (Gao et al., 2013), yellow croaker (Pseudosciaena crocea) (Mu et al., 2010), and striped murrel (Channa striatus) (Kumaresan et al., 2016). Casp3 has been investigated in sea bass (Reis et al., 2007b), zebrafish (Danio rerio) (Yabu et al., 2001), Atlantic salmon (Salmo salar) (Takle et al., 2006), yellow croaker (Li et al., 2011) and striped murrel (Kumaresan et al., 2016), but two Casp3 subtypes (Casp3a and Casp3b) were characterized only in Atlantic salmon (Takle et al., 2006) and medaka Oryzias latipes (Naruse et al., 2000). To the best of our knowledge, the sequence information of Cycs and Apaf1 genes still remains unknown in fish. In addition, limited studies explore their constitutive tissue expression profiles in fish, such as Casp9 in sea bass (Reis et al., 2007a) and large yellow croaker (Mu et al., 2010), Casp3 in large yellow croaker (Li et al., 2011).

Apoptotic occurrence can be influenced by dietary composition (Tie et al., 2019; Ye et al., 2019; Yin et al., 2019). Dietary fat plays an important role in providing energy, essential fatty acids (EFAs) and fat-soluble vitamins (Luo et al., 2005), especially for fish, as they have limited abilities in utilizing carbohydrates as an energy source. However, high dietary fat levels is harmful for fish growth and health (Luo et al., 2005). Using mammals and their cell lines, several studies report that HFDs influenced the process of apoptosis, associated with the disruption of mitochondrial function (Moraes et al., 2009). However, in fish, studies involved in the effect of HFD on expression of apoptosis related genes are very scarce.

Yellow catfish Pelteobagrus fulvidraco, a freshwater omnivorous teleost, was widely cultured in several Asian countries due to its good filet quality and high market value. However, under intensive aquaculture, dietary fat levels often amount to $10.0 \%$, which inevitably leads to excess fat deposition in the livers and visceral tissues of yellow catfish. Excessive fat deposition will reduce growth performance and a healthy status for this fish species. Since apoptosis is a highly regulated form of programmed cell death and related to a healthy status for organisms, the present working hypothesis is that HFD can influence mRNA expression of genes involved in the mitochondrial apoptotic pathway. To this end, in this study, the full-length cDNA sequences of Cycs, Apaf1, Casp9, Casp3a and Casp $3 \mathrm{~b}$ were cloned and characterized, and their tissue-specific expressions were explored from yellow catfish P. fulvidraco, a widely distributed freshwater omnivorous teleost in several Asian countries. The transcriptional responses of Cycs, Apaf1, Casp9, Casp3a, and Casp3b genes to HFD were then investigated in various tissues of $P$. fulvidraco.

\section{MATERIALS AND METHODS}

Two experiments were conducted. Experiment 1 was conducted to clone the full-length cDNA sequences of Cycs, Apaf1, Casp9, Casp3a and Casp3b, and to investigate their mRNA tissue expression profiles. Experiment 2 evaluated the changes of mRNA levels of Cycs, Apaf1, Casp9, Casp3a, and Casp3b of various tissues in yellow catfish to HFD. We ensured that the experiments performed on animals followed the ethical guidelines of Huazhong Agricultural University and confirm that all experimental protocols were approved by Huazhong Agriculture University.

\section{Experiment 1: Cloning of Cycs, Apaf1, Casp9, Casp3a, and Casp3b Genes and Exploring Their mRNA Tissue Expression}

Yellow catfish P. fulvidraco (initial body weight: $16.5 \pm 3.4 \mathrm{~g}$, mean \pm SEM) were obtained from a local commercial farm, Wuhan, China, and stocked in indoor fiberglass tanks. The culture protocols were similar to those described in our recent publication (Wei et al., 2017). Briefly, yellow catfish (initial body weight: $16.5 \pm 3.4 \mathrm{~g}$, mean \pm SEM) were maintained in indoor cylindrical fiberglass tanks (300 L water volume) for a 2 week acclimation. All fish were fed a commercial pellet diet with a fat level of $9.7 \%$, twice a day. They were provided with continuous aeration to maintain the dissolved oxygen level near saturation. At the end of the 2 week acclimation, fish were fasted for $24 \mathrm{~h}$ and then euthanized with MS-222 (100 mg/L). Liver, muscle, spleen, intestine, gill, mesenteric fat, heart, kidney, and ovary tissues were quickly collected and frozen in liquid nitrogen and stored at $-80^{\circ} \mathrm{C}$ for RNA isolation. RNA isolation, synthesis of cDNAs and cloning of Cycs, Apaf1, Casp9, Casp3a, Casp3b genes were based on the protocols described in our studies (Wei et al., 2017). The quality of total RNA was checked by agarose gel electrophoresis. The concentration and the ratio of $\mathrm{OD}_{260} / \mathrm{OD}_{280}$ of total RNA were determined using a Nanodrop ND-2000 spectrophotometer (Thermo Fisher Scientific, United States) for the determination of $\mathrm{OD}_{260}$, $\mathrm{OD}_{280}$ and $\mathrm{OD}_{230}\left(\mathrm{OD}_{260} / \mathrm{OD}_{280}>1.8, \mathrm{OD}_{260} / \mathrm{OD}_{230}>1.5\right)$. Degenerate primers (Supplementary Table 1), designed based on the most conserved regions of these fish Cycs, Apaf1, Casp9, Casp3a, and Casp3b sequences available in the GenBank and Ensembl database, were used to amplify partial cDNA fragments. The $3^{\prime}$ and $5^{\prime}$ end sequences were obtained through nested $3^{\prime}$ and $5^{\prime}$ RACE PCR performed with a SMART RACE cDNA Amplification Kit (Clontech, United States) based on the manufacturer's manual. 
The edition of assembled full-length sequences, sequence alignments and percentage of amino acid conservation were similar to those described in our recent publication (Wei et al., 2017). Domains were analyzed by the SMART program ${ }^{1}$ and an online $\mathrm{CDD}$ tool at $\mathrm{NCBI}^{2}$. The phylogenetic trees were generated through a neighbor-joining (NJ) method with MEGA 5.0 (Tamura et al., 2011) based on the JTT $+\mathrm{G}$ model (Jones et al., 1992), and the best-fit model of sequence evolution was obtained by ML model selection. Bootstrap sampling was reiterated 1000 times.

\section{Experiment 2: Transcriptional Responses of Cycs, Apaf1, Casp9, Casp3a, and Casp3b of Various Tissues of Yellow Catfish Fed HFDs}

Two experimental diets were formulated with dietary fish oil/soyoil $(1: 1, w / w)$ supplemented at fat levels of $6 \%$ (control fat) and $10 \%$ (HFD). Final fat levels were determined to be 11.34 and $15.41 \%$ for the control and HFD, respectively. When beginning the feeding experiment, 30 uniform-sized fish (mean initial weight: $3.79 \pm 0.16 \mathrm{~g}$ ) were randomly stocked in each fiberglass tank. Two diet (control and HFD) was assigned to six tanks in a completely randomized design, three replicates for each diet. The fish were fed to apparent satiation twice daily at two equal meals (9:00 and 16:00 h) during the week. The experiment continued for 8 weeks.

At the termination of the feeding study, all fish were fasted for $24 \mathrm{~h}$. They were then anesthetized with tricaine methane sulfonate (MS-222 at $100 \mathrm{mg} / \mathrm{L}$ ). Three fish per aquarium were collected randomly, and mesenteric fat, intestine, ovary, kidney, heart, spleen and gill tissues were isolated and quickly frozen in liquid nitrogen, and reserved at $-80^{\circ} \mathrm{C}$ for the subsequent analysis for quantitative PCR.

\section{qPCR Determination}

The mRNA levels were assayed by the real-time qPCR method described in our recent publication (Wei et al., 2017). The primer sequences of each gene used in this analysis are given in Supplementary Table 2. The qPCR program included $1 \mathrm{~min}$ at $95^{\circ} \mathrm{C}$ and 40 cycles at $95^{\circ} \mathrm{C}$ for $5 \mathrm{~s}, 60^{\circ} \mathrm{C}$ for $10 \mathrm{~s}$, and $72^{\circ} \mathrm{C}$ for $30 \mathrm{~s}$. All reactions were performed in duplicate, and each reaction mixture was checked to ensure that it contained a single product of the correct size by agarose gel electrophoresis. As housekeeping gene sequences were not available in $P$. fulvidraco, a set of eight housekeeping genes (18S rRNA, RPL7, $\beta$-actin, HPRT, TUBA, B2M, TBP, GAPDH, ELFA, and UBCE) were selected from the literature (Zhao et al., 2011) in order to test their transcription stability. For each control gene, we determined the pairwise variation with all other control genes as the standard deviation of the logarithmically transformed expression ratios and defined the internal control gene stability by measuring the $M$ value as the average pairwise variation of a particular gene with all the other control genes. Genes with the lowest $M$ values

${ }^{1}$ http://www.smart.embl-heidelberg.de/

${ }^{2}$ http://www.ncbi.nlm.nih.gov/Structure/cdd/wrpsb.cgi have the most stable expression. The relative expression of genes was calculated using the $2^{-\Delta \Delta \mathrm{Ct}}$ method (Livak and Schmittgen, 2001) normalizing to the geometric mean of the best combination of two genes as suggested by geNorm (Vandesompele et al., 2002). Prior to the analysis, experiments were performed to check the stability of housekeeping genes, from which GAPDH and HPRT, $\beta$-actin and UBCE showed the most stable level of expression in tissues distribution analysis experiment and HFD experiment, respectively, across the experimental conditions.

\section{Statistical Analysis}

Results are presented as mean \pm SEM. Prior to statistical analysis, all data were tested for normality of distribution using the Kolmogorov-Smirnov test. The results of the homogeneity of variances among the different tissues were then determined by one-way analysis of variance (ANOVA) and Tukey's multiple range test. Differences between the control and HFD group were analyzed by Student's $t$-test for independent samples. The analysis was carried out using the SPSS 20.0 for Windows (SPSS, Michigan Avenue, Chicago, IL, United States), and the minimum significant level was set at 0.05 .

\section{RESULTS}

\section{cDNA Cloning and Sequence Analyses of Five Genes}

Using RT-PCR and $3^{\prime}$ - and 5'-RACE PCR, we successfully cloned the full-length cDNA sequences of Cycs, Apaf1, Casp9, Casp3a, and Casp3b. The validated cDNA sequences of P. fulvidraco cytochrome c (Pf-Cycs, GenBank Accession No. KY053836), P. fulvidraco Apaf1 (Pf-Apaf1, GenBank Accession No. KY053839), P. fulvidraco caspase 9 (Pf-Casp3b, GenBank Accession No. KY053837), P. fulvidraco caspase 3a (Pf-Casp3a, GenBank Accession No. KY072821), P. fulvidraco caspase 3b (Pf-Casp3b, GenBank Accession No. KY072822) were 754, 5028, 1544, 1831, and 1225 bp in length, encoding the peptides of $104,1259,442,284$, and 285 amino acid residues, respectively (Table $\mathbf{1}$ ).

The pair-wise amino acid sequence comparison of five genes between different species are shown in Table 2. The Cycs in $P$. fulvidraco shared high identity (83.7-99\%) with the corresponding Cycs orthologous genes from other fish species, amphibians and mammals. The amino acid sequences of P. fulvidraco Cycs, Apaf1, caspase 9, caspase 3a, and caspase 3b

TABLE 1 | The sequence information of five apoptosis related genes from P. fulvidraco.

\begin{tabular}{lcccccc}
\hline Genes & $\begin{array}{c}\text { Accession } \\
\text { No. }\end{array}$ & $\begin{array}{c}\mathbf{5}^{\prime} \text { UTR } \\
\mathbf{( b p )}\end{array}$ & $\begin{array}{c}\text { ORF } \\
\mathbf{( b p )}\end{array}$ & $\begin{array}{c}\mathbf{3}^{\prime} \text { UTR } \\
\mathbf{( b p )}\end{array}$ & $\begin{array}{c}\text { Full length } \\
\mathbf{( b p )}\end{array}$ & $\begin{array}{c}\text { Protein } \\
\text { (aa) }\end{array}$ \\
\hline Cycs & KY053836 & 81 & 315 & 358 & 754 & 104 \\
Apaf1 & KY053839 & 127 & 3780 & 1121 & 5028 & 1259 \\
Casp9 & KY053837 & 33 & 1329 & 182 & 1544 & 442 \\
Casp3a & KY072821 & 219 & 855 & 757 & 1831 & 284 \\
Casp3b & KY072822 & 86 & 858 & 281 & 1225 & 285
\end{tabular}


TABLE 2 | Amino acid sequence identity of five apoptosis related genes between P. fulvidraco and other species (\%).

\begin{tabular}{lccccc}
\hline Genes & $\begin{array}{c}\text { Ictalurus } \\
\text { punctatus }\end{array}$ & $\begin{array}{c}\text { Danio } \\
\text { rerio }\end{array}$ & $\begin{array}{c}\text { Xenopus } \\
\text { tropicalis }\end{array}$ & $\begin{array}{c}\text { Mus } \\
\text { musculus }\end{array}$ & $\begin{array}{c}\text { Homo } \\
\text { sapiens }\end{array}$ \\
\hline Cycs & 99 & 99 & 87.5 & 90.4 & 83.7 \\
Apaf1 & 88.3 & 76.5 & 51.2 & 56 & 56.5 \\
Casp9 & 83.3 & 72.4 & 38.1 & 48.5 & 48.4 \\
Casp3a & 85.5 & 74.5 & 57.2 & 58.5 & 57.7 \\
Casp3b & 77.9 & 65.8 & 55.1 & 59.4 & 56.9 \\
\hline
\end{tabular}

Accession numbers as follows (the order is Ictalurus punctatus, Danio rerio, Xenopus tropicalis, Mus musculus, and Homo sapiens): Cycs (XP_017338026.1, NM_001002068.1, ENSXETP00000049267, JF919281.1, NM_018947.5); Apaf1 (XM_017485104, NM_131608.1, XM_012959843.2, NM_001042558.1, ENSP00000448165); Casp9 (XM_017487601, NM_001007404.2, NM_001123463, XM_017319928.1, ENSP00000330237); Casp3a (XP_017317002, NM_131877.3, NM_001127428.1, NM_001284409.1, XM_011532301.1); Casp3b (NM_001201081, XM_005173075.4, NM_001127428.1,NM_001284409.1, XM_011532301.1).

were similar to those from other fish and mammals, exhibiting $51.2-88.3,38.1-83.3,57.2-85.5$, and 55.1-77.9\% amino acid sequence identities, respectively.

The protein sequences of $P$. fulvidraco Cycs possessed highly conserved Cycs domain, including several interactive sites with heme and iron ion and one modified site of trimethyllysine, composing of five $\alpha$-helices $(\alpha 1-\alpha 5)$ (Figure 1). The P. fulvidraco Apaf1 embraced all the characteristic features of Apaf1, including the CED-3 and CED-4 homologous regions, the seven short $\alpha$-helices identified in Apaf1 CARD, the Walker's A- and B-box consensus sequences for nucleotide binding sites, and the WD repeat motifs are indicated (Figure 2 ). The predicted amino sequence of Casp9 contained a similar architecture than mammals, including a prodomain, a large subunit, a small subunit, the putative cleavage sites, a caspase recruitment domain (CARD), consensus and putative Akt phosphorylation motifs, A-X-P-X motifs, caspase family histidine and cysteine active sites along with the conserved active residues 'His' and 'Cys.' Furthermore, the conserved 'Arg' and 'Leu' residues in prodomain and the conserved 'Tyr' were also found in P. fulvidraco (Figure 3). Casp3 (Casp3a and 3b) contained a similar structure with mammals, including a prodomain, a large subunit, a small subunit, the putative cleavage sites, and cysteine active sites. Several residues known to be critical in the Casp3 catalytic and binding pocket were found in P. fulvidraco. The two tryptophan residues and tyrosine residues were also found in P. fulvidraco and other vertebrates (Figure 4). In Casp9, two putative cleavage sites at aspartic acid residues separates the large subunit and small subunit, while the large subunit is contiguous with the small subunit in Casp3.

\section{Phylogenetic Analysis}

A phylogenetic analysis based on the amino acid sequences of Cycs, Apaf1, Casp9, Casp3a, and Casp3b from P. fulvidraco and other vertebrate species are shown in Figure 5. According to the phylogenetic analysis, all teleost cytochrome $\mathrm{c}$ formed an independent cluster, while amphibian and mammalian cytochrome c formed another cluster. P. fulvidraco Cycs were grouped with Astyanax mexicanus and Danio rerio, the same members of Ostariophysi. They then formed a clade with the species of Acanthomorphata (Takifugu rubripes, Oryzias latipes, Xiphophorus maculatus, Oreochromis niloticus). A similar topology was also observed in phylogenetic trees of Apaf-1. The phylogenetic analysis showed that all Casp3 and Casp9 genes clustered with the corresponding sequences of vertebrates. Casp3 clades together constituted the caspase 3 subfamily, which is clearly separated from Casp9 clade. All teleost Casp3a and Casp3b formed a cluster, whereas mammalian Casp3 formed another clade. Moreover, on the basis of the tree topologies, there appeared to be a fish-specific gene duplication which may result

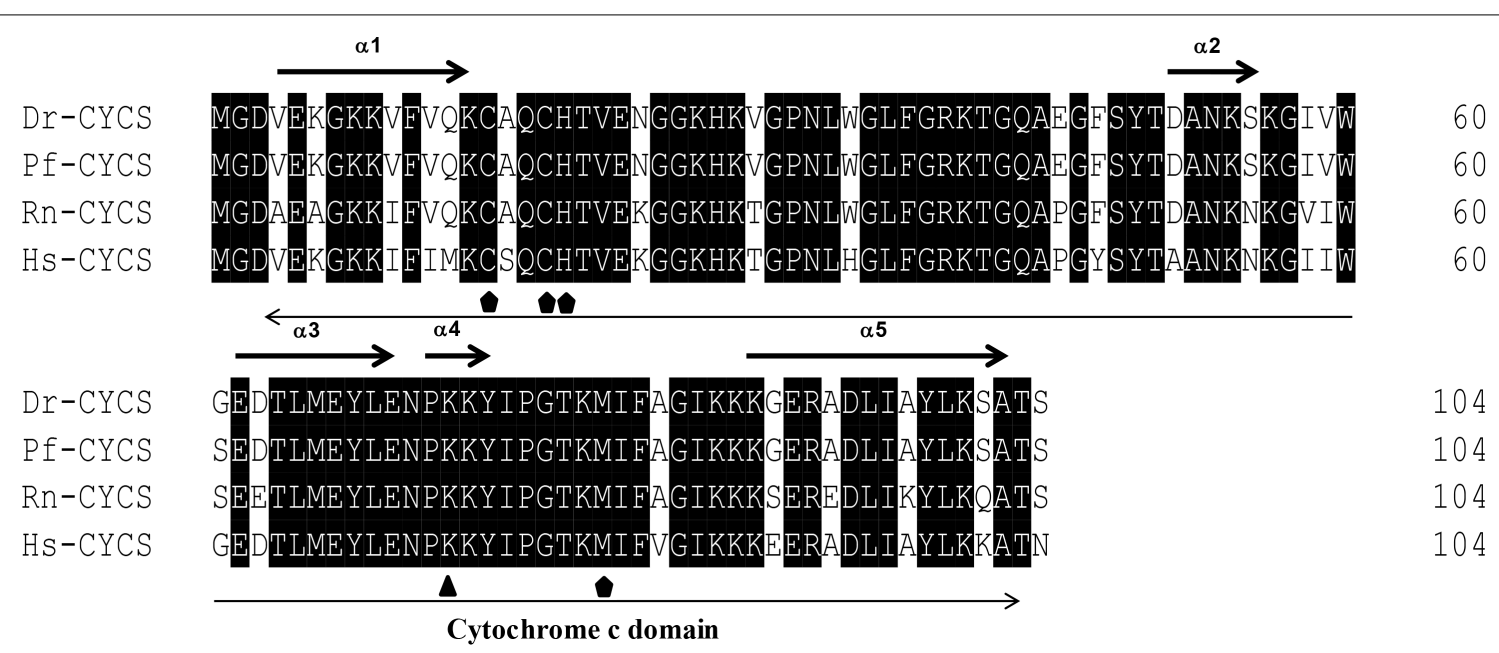

FIGURE 1 | Multiple amino acid sequence alignments of Cycs genes of Pelteobagrus fulvidraco and other species. Accession numbers are ENSRNOP00000041521, NM_018947.5, NM_001002068.1, KY053836 for Rattus norvegicus (Rn), Homo sapiens (Hs), Danio rerio (Dr), and Pelteobagrus fulvidraco (Pf), respectively. Arrows above and below the sequences represent $\alpha$-helices and the Cytochrome c domain, respectively. Residues below symbols ( were identified as a specific site, including the interactive sites with heme and iron ion. Residues below symbol $(\boldsymbol{\Lambda})$ means the modified sites of trimethyllysine. 


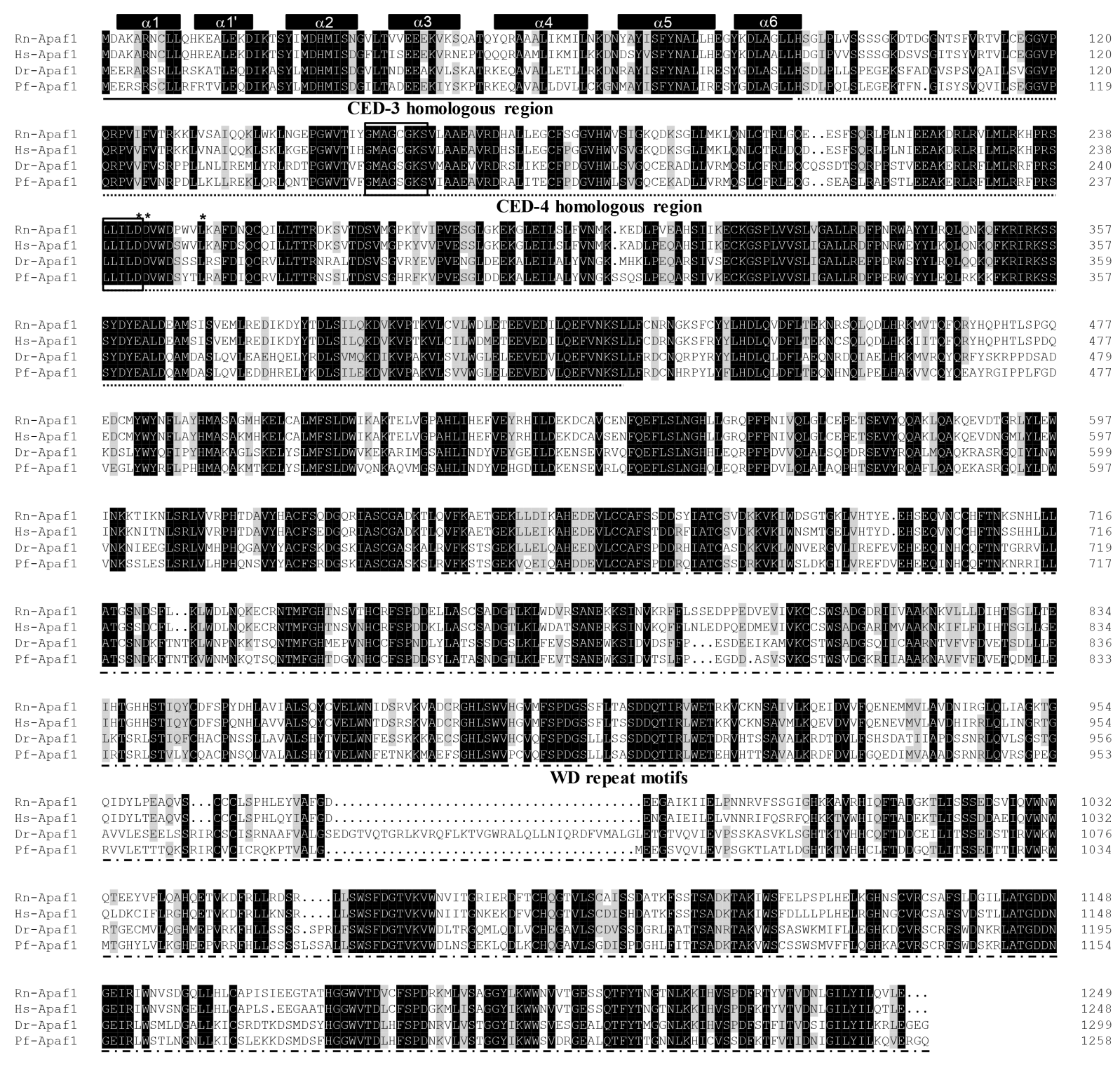

FIGURE 2 | Multiple amino acid sequence alignment of Apaf1 genes from P. fulvidraco and other species. Accession numbers were ENSRNOP00000041521, ENSP00000448165, NM_131608.1, KY053839 for Rattus norvegicus (Rn), Homo sapiens (Hs), Danio rerio (Dr), and Pelteobagrus fulvidraco (Pf), respectively. The CED-3 and CED-4 homologous region and WD repeat motifs indicate in a continuous and discontinuous line. Apaf1 CARD (caspase recruitment domain) contains seven short $\alpha$-helices which are represented by cylinders on top of the sequence alignment. The Walker's A- and B-box consensus sequences for nucleotide binding sites at amino acid residues 153-160 and 238-242 are boxed. Asterisks denote the amino acid residues that are known to be important for CED-4 function. Deep and light shadows represent homology of 100 and $75 \%$, respectively.

in the generation of an additional Casp3 member in P. fulvidraco and other fishes (Casp3a and Casp3b).

\section{mRNA Expression Patterns of Cycs, Apaf1, Casp9, Casp3a, and Casp3b Genes Among Nine Tissues}

The Cycs mRNA was the highest in ovary tissues, followed by the heart, gill, muscle, and spleen tissues, but lowest in the kidney, liver, mesenteric fat, and intestine tissues (Figure 6A). The mRNA of Apaf1 was predominantly expressed in spleen and kidney tissues, followed by gill tissues, and lowest in heart, intestine, mesenteric fat, muscle, liver, and ovary tissues (Figure 6B). The mRNA of Casp9 was highest in spleen tissues, followed by ovary, gill, mesenteric fat, kidney, intestine, liver tissues, and the lowest in the heart and muscle tissues (Figure 6C). The Casp3a mRNA expression was predominant in spleen tissues, followed by gill, ovary and heart tissues, and the lowest in mesenteric fat, muscle, kidney, intestine, and the liver tissues (Figure 6D). For the Casp3b mRNA levels, the values were highest in the spleen tissues, followed by gill, mesenteric fat, intestine and the 


\section{Prodomain}

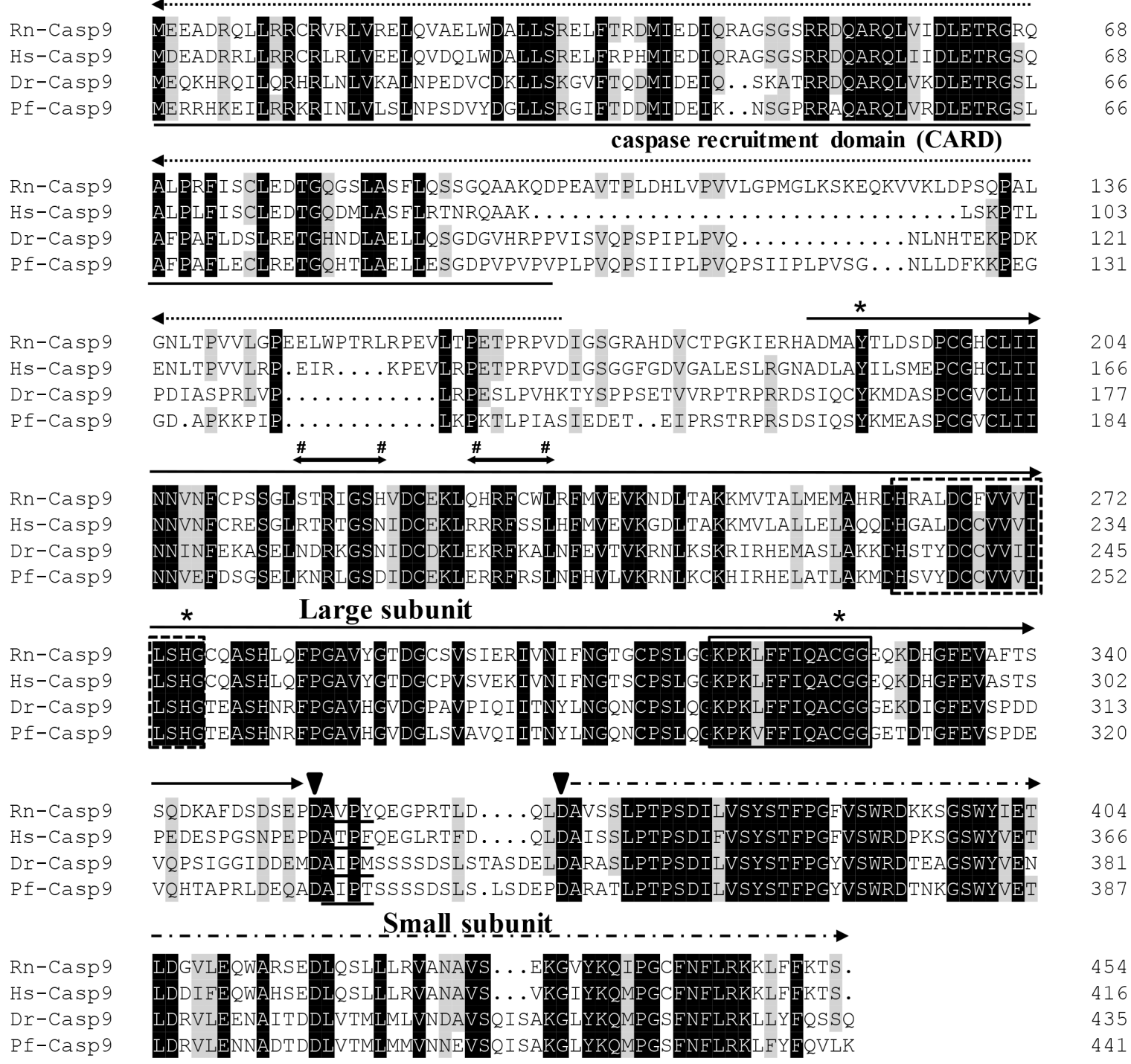

FIGURE 3 | Multiple amino acid sequence alignments of Casp9 genes from P. fulvidraco and other species. Accession numbers as follows [the order is Rattus norvegicus (Rn), Homo sapiens (Hs), Danio rerio (Dr), and Pelteobagrus fulvidraco (Pf)]: Casp9 (ENSRNOP00000017972, ENSP00000330237, NM_001007404.2, KY053837). The putative cleavage site at aspartic acid residues (Asp333 and Asp351), which separates the large subunit $(\rightarrow$ ) from the small subunit $(-\rightarrow)$ are indicated by arrowheads. The prodomain is indicated by a dashed line with an arrowhead (4....). The caspase family histidine (residues 242-256) and cysteine active sites (residues 296-307) are boxed in a discontinuous or continuous line, respectively. The first 100 amino acids (caspase recruitment domain, underlined) are a putative $\mathrm{N}$-terminal CARD which are presumably required to bind to Apaf1. Residues below symbols (\#) indicate the consensus and putative Akt phosphorylation motifs. The A-X-P-X motifs are underlined. The conserved 'Tyr,' 'His,' and 'Cys' residues are indicated with an asterisk (*). The prodomain regions comprise conserved 'Arg' and 'Leu' residues. Deep and light shadows represent homology of 100 and $75 \%$, respectively.

kidney tissues, and showed no significant differences in other tissues (Figure 6E).

\section{Transcriptional Responses of Cycs, Apaf1, Casp9, Casp3a, and Casp3b of Various Tissues in Yellow Catfish Fed HFDs}

In the mesenteric fat tissues, compared to the control, a HFD up-regulated the mRNA expression of Cycs and Casp3a and had no significant effect on the expression of Apaf1, Casp9, and Casp3b (Figure 7A); in the intestine tissues, a HFD upregulated the expressions of Cycs, Casp9, Casp3a and Casp3b, but Apaf1 expression showed no significant change (Figure 7B). In ovary tissues, a HFD upregulated the mRNA expression of Apaf1, Casp9, Casp3a and Casp3b significantly, and the expression of Cycs remained relatively constant (Figure 7C). In kidney tissues, a HFD significantly upregulated the mRNA expression of Apaf1, Casp9 and Casp3a, but did not significantly influence the expression Cycs and Casp3b (Figure 7D). 


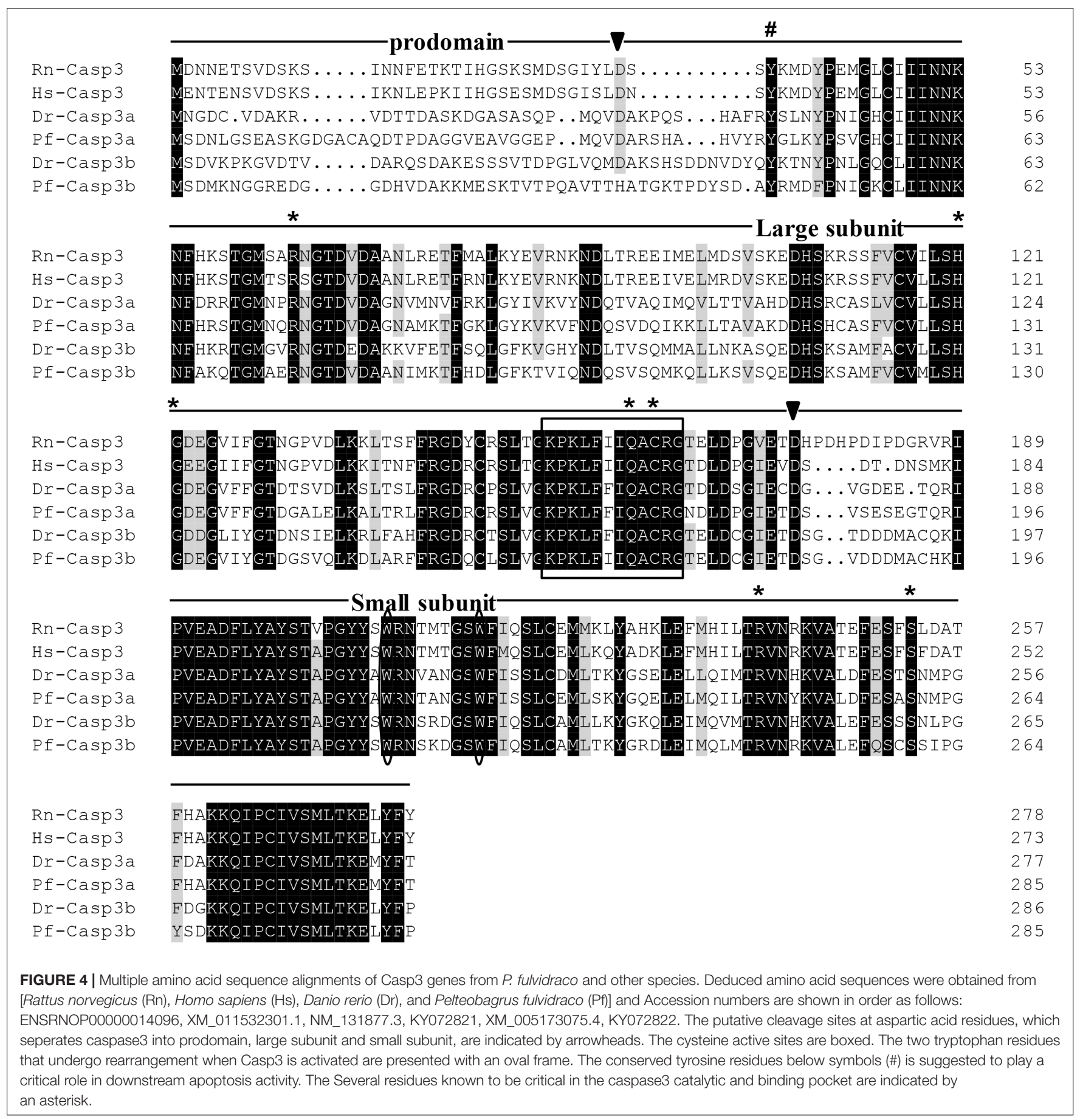

In heart tissues, compared with the control, a HFD downregulated the mRNA expression of Cycs, Apaf1, Casp9 and Casp3b, but did not significantly influence the expression of Casp3a (Figure 7E); in spleen tissues, the expression of Cycs, Apaf1, Casp9, Casp3a, and Casp3b were down-regulated in a HFD (Figure 7F). In gill tissues, a HFD significantly down regulated the mRNA expressions of Apaf1, Casp9, Casp3a and Casp3b, but did not significantly influence Cycs mRNA expression (Figure 7G).

\section{DISCUSSION}

In the present study, we successfully cloned the full-length cDNA sequences of Cycs, Apaf1, Casp9, Casp3a, and Casp3b from $P$. fulvidraco. To the best of our knowledge, this is the first report involving cDNA sequence information and mRNA tissue expression profiles of Cycs and Apaf1, and their transcription responses of the five genes to a HFD in fish. 


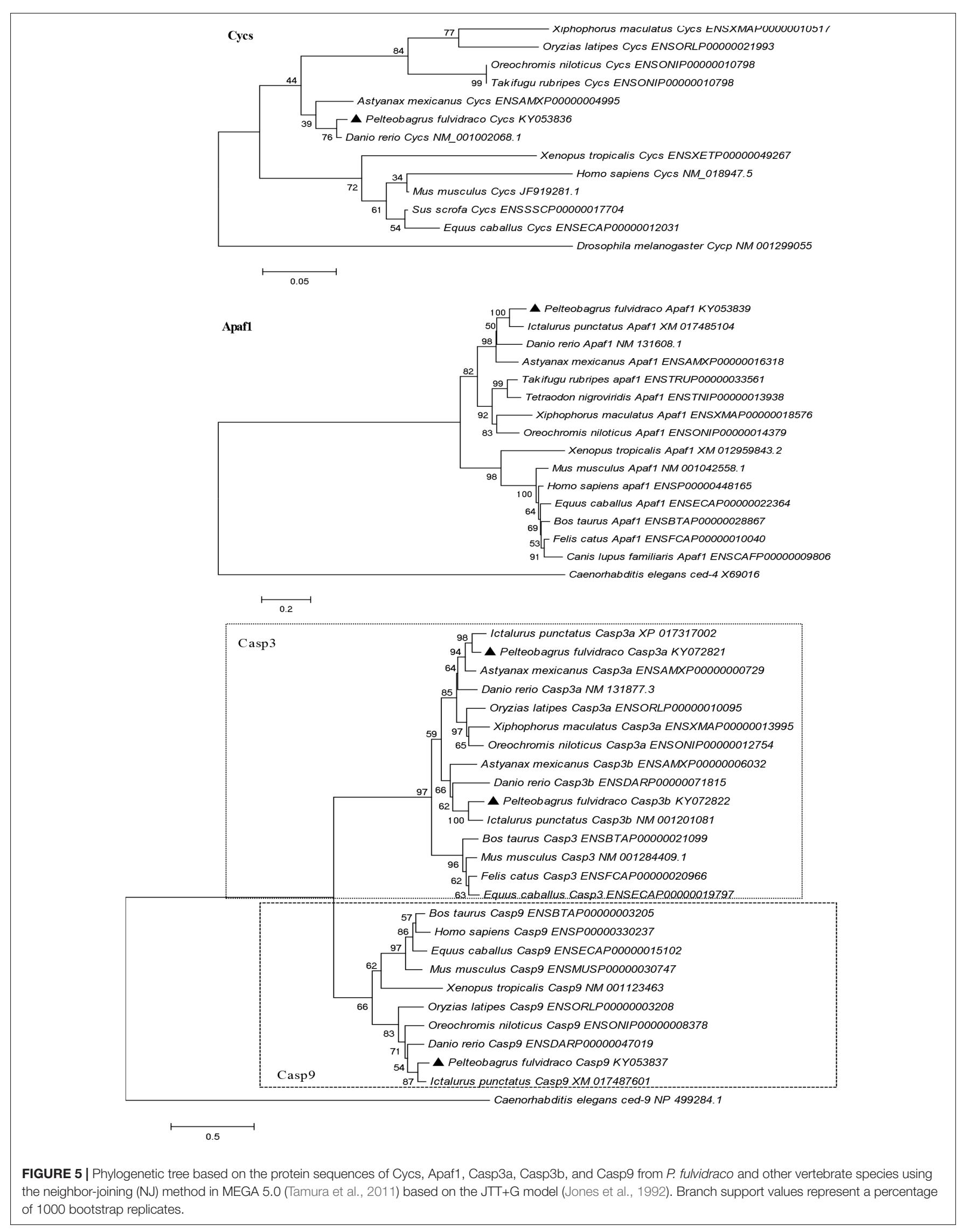


A

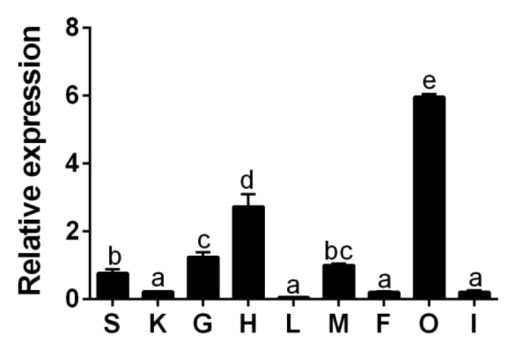

C

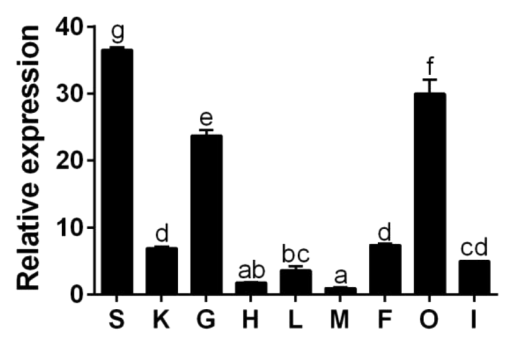

E

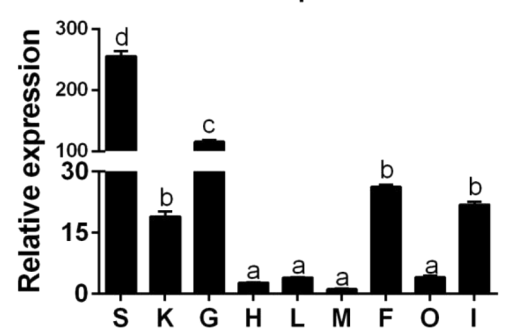

B

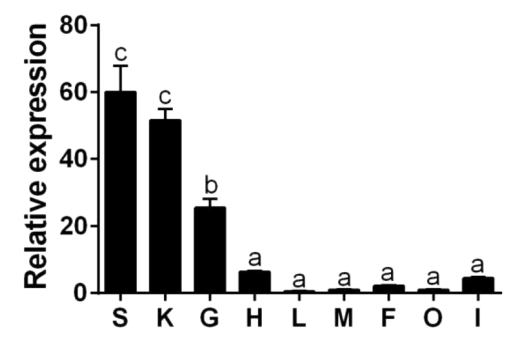

D

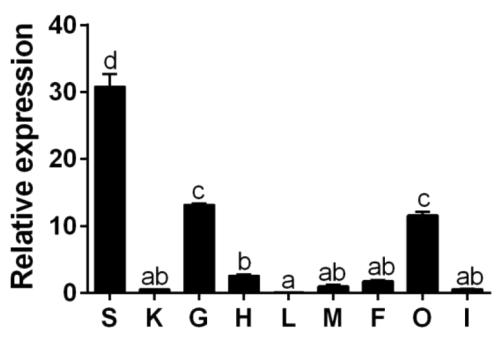

FIGURE 6 | The tissue-specific expression of five apoptosis related genes across spleen (S), kidney (K), gill $(G)$, heart $(H)$, liver $(L)$, muscle $(M)$, mesenteric fat $(F)$, ovary (O), and intestine (I) of P. fulvidraco via quantitative PCR (Q-PCR) (A: Cycs; B: Apaf1; C: Casp9; D: Casp3a; E: Casp3b). Data (mean \pm SEM, $n=6)$ were expressed relative to expression of housekeeping gene (GAPDH and HPRT). Expression of genes in muscle was regarded as the relative expression 1. Bars with different letters indicate significant differences among nine tissues $(P<0.05)$.

Our study indicated that the putative protein sequences of $P$. fulvidraco Cycs and Apaf1 embraced all the characteristic features of their corresponding parts in mammals, indicating that they might have a similar function to those in mammals. The predicted amino acid sequence of caspase 9 contained a putative CARD motif, followed by the large (p20) and small (p10) subunit, in agreement with other studies (Gao et al., 2013; Kumaresan et al., 2016). Furthermore, the conserved 'Arg' and 'Leu' residues in prodomain and the conserved 'Tyr', as reported by Kumaresan et al. (2016), were also found in P. fulvidraco. The characteristic Casp9 pentapeptide active-site QACGG is conserved in other fish (Gao et al., 2013) and also located in the large subunit (Reis et al., 2007a). In the present study, the deduced Casp3 (Casp3a and 3b) in yellow catfish is highly homologous with the Casp3 of other species, including a putative prodomain followed by a large and a small subunit (Yabu et al., 2001; Reis et al., 2007b; Li et al., 2011). The Casp3 sequence retains the motifs that are functionally important, such as the pentapeptide active-site motif (QACRG) and the putative cleavage sites at the aspartic acids, in agreement with those in large yellow croaker and sea bass (Reis et al., 2007b; Li et al., 2011). In addition, the present study also cloned two Casp3 isoforms in yellow catfish, in agreement with reports in medaka Oryzias latipes (Naruse et al., 2000) and Atlantic salmon (Takle et al., 2006). However, in other fish species, the Casp3 gene exists as a single copy gene (Reis et al., 2007b; Li et al., 2011).

The successful cloning of the five key genes enabled us to study their constitutive and diet-induced tissue expression profiles. However, to the best of our knowledge, this is the first report involved in mRNA constitutive tissue expression profiles of Cycs, Apaf1 and Casp3b in fish, which limited our comparison with other fish. The present study showed that mRNAs of the five genes were constitutively expressed in all tested tissues, indicating that they participated in many physiological functions in these tissues. However, their mRNA expression varied with the tissues, indicating their tissue-specific roles. On the other hand, we found that the Cycs mRNA was the highest in ovary tissues, followed by heart, gill, muscle and spleen tissues, but lowest in kidney, liver, mesenteric fat, and intestine tissues. In shrimp, 


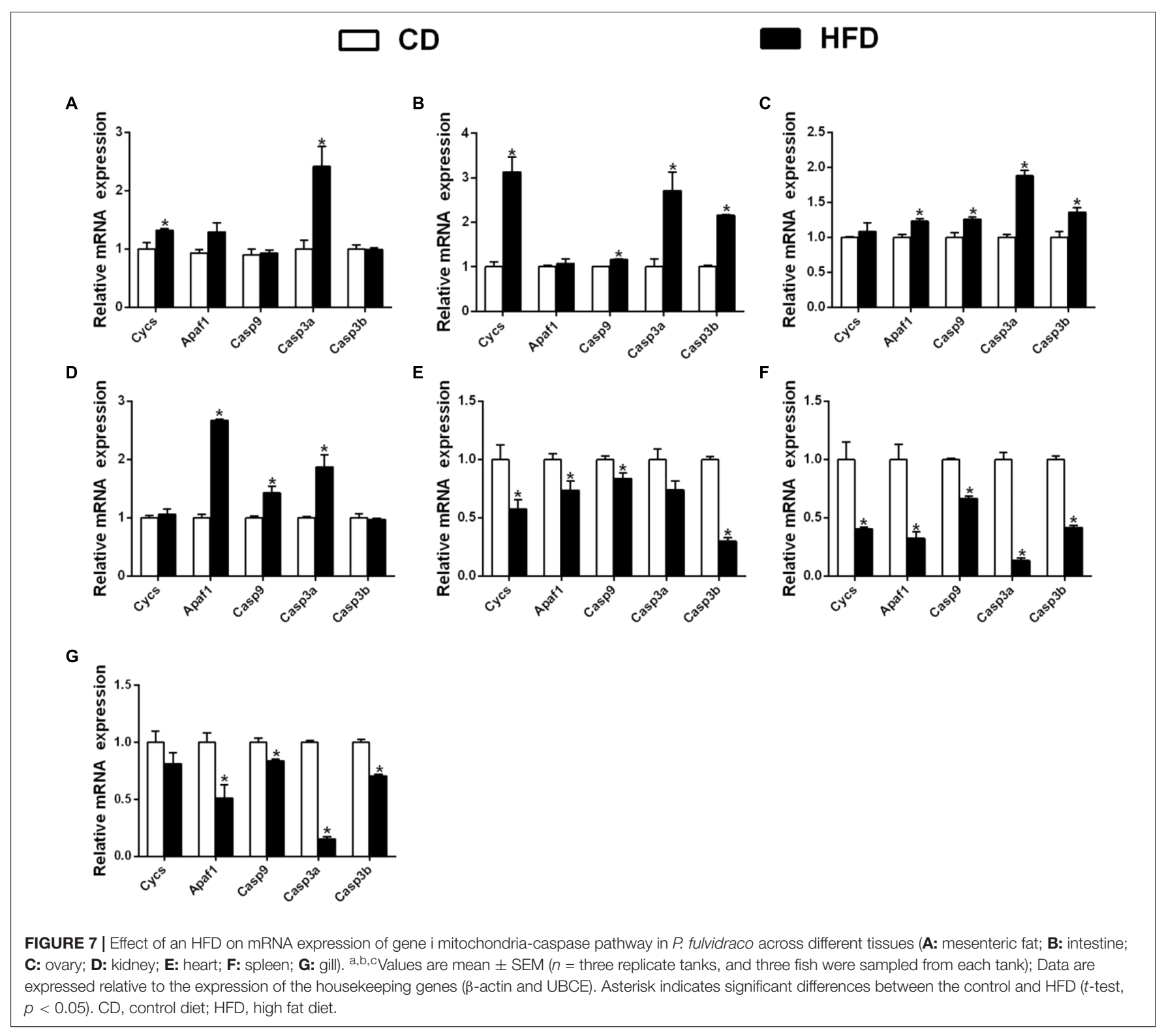

$\mathrm{Hu}$ and Yao (2016) reported the ubiquitous expression of Cycs in most examined tissues of shrimp Litopenaeus vannamei, but predominantly in muscle tissues, followed by intestine, gill, and heart tissues. The present study indicated that the mRNA of Apaf1 was predominantly expressed in spleen and kidney tissues, followed by gill, and lowest in heart, intestine, mesenteric fat, muscle, liver, and ovary tissues. In mammals, Walke and Morgan (2000) reported that Apaf1 transcript was detected in many tissues, and the highest level of expression was seen in spleen and lung tissues. We also found that the mRNA of Casp9 was highest in spleen tissues, followed by ovary, gill, mesenteric fat, kidney, intestine, liver, and the lowest in heart and muscle tissues, in partial agreement with several other studies. For example, $\mathrm{Mu}$ et al. (2010) pointed out that large yellow croaker Casp9 was constitutively expressed in all tissues examined with heart tissues containing the highest levels, and intestine and muscle tissues containing the lowest levels. In sea bass, Reis et al. (2007a) observed the highest expression levels of Casp9 in heart and liver tissues, and low expression in spleen and kidney tissues. Kumaresan et al. (2016) reported that Casp9 was highly expressed in trunk kidney tissues followed by head kidney tissues of C. striatus. The present study found that two Casp3 subtypes, were differentially expressed in various tissues. For example, the Casp3a mRNA expression was predominant in spleen tissues, followed by gill, ovary and heart tissues, the lowest in mesenteric fat, muscle, kidney, intestine, and liver tissues; for the Casp3b mRNA levels, the values were highest in spleen tissues, followed by gill, mesenteric fat, intestine and kidney tissues, and showed no significant differences in other tissues. The differences in expression patterns among yellow catfish caspases suggested that each caspase might differ functionally, as suggested by Takle et al. (2006). In large yellow croaker, Li et al. (2011) reported that Casp3 
was constitutively expressed in various tissues, with higher levels in blood, heart, kidney, spleen, intestine and gill tissues, but lower levels in liver and muscle tissues. In C. striatus, the maximum expression of Casp3 is observed in spleen tissues (Kumaresan et al., 2016). In contrast, in sea bass, Casp3 mRNA was expressed in all tissues examined, albeit at low levels (Reis et al., 2007b).

In fish, the dynamic mRNA expression of apoptosis-related genes has been used as a marker at an earlier point in the apoptotic cascade (Gao et al., 2013), and their increased mRNA expression has been described in cells under apoptosis (Chiang et al., 2001; Yakovlev et al., 2001). The present study indicated that effects of a HFD on the mRNA expression of genes in intrinsic mitochondria pathways were tissue dependent. Generally speaking, two trends were observed: in the mesenteric fat, intestine, ovary and kidney tissues, a HFD induced mRNA levels of several genes among these five key genes; however, in the heart, spleen and gill tissues, a HFD down-regulated mRNA expression of partial genes among these five key genes. At present, we do not know the exact reason, since this is the first study exploring the changes of their mRNA expression of genes involved in the mitochondrial apoptotic cascade of various tissues in fish fed an HFD. Apoptosis is one of major types of cell death, and previous studies demonstrate that diets could influence the expression of apoptotic genes. For example, Camargo et al. (2017) showed that long-term consumption of low-fat, high-complex carbohydrate diets (LFHCC) and LFHCC $\mathrm{n}-3$ diets increased the expression of Casp3 in the adipose tissue. Olivo and Hilakivi-Clarke (2005) showed that an HFD inhibited apoptosis in rat mammary glands. An HFD induced apoptosis in skeletal muscle (Sishi et al., 2011) and liver tissues (Wang et al., 2008). Moraes et al. (2009) observed that an HFD induced the hypothalamic expression of Apaf1 and Casp9. In livers of rats fed HFD compared to those in the control, there was higher levels of cleaved Casp3 (Wang et al., 2008). Espe et al. (2015) pointed out that enhanced activation of caspase 3 was associated with the oxidative stress from Atlantic salmon. Li et al. (2017) found that $\mathrm{Zn}$ increased hepatic transcriptional levels of cytochrome $\mathrm{C}$, caspase $3 \mathrm{a}$ and caspase $3 \mathrm{~b}$ in yellow catfish, which indicated that $\mathrm{Zn}$ induced mitochondrial-mediated apoptosis. Yin et al. (2019) found that the expression of caspase 9 significantly increased with an increasing oxidative degree of fish oil in largemouth bass. Thus, the reduced apoptotic mRNA levels in the HFD group in heart, spleen, and gill tissues will inhibit apoptosis via mitochondrial apoptotic pathways, which may result in the impairment of the capacity for regeneration and repair of these tissues and organs, as suggested by Ye et al. (2019). Increased mRNA levels of these apoptotic genes in mesenteric fat, intestine, ovary, and kidney tissues indicate that an HFD activates the mitochondrial apoptotic pathway and induces apoptosis in these organs, as suggested by Li et al. (2017). Therefore, our result indicates that an HFD differentially influences mRNA expression of the genes of various tissues in intrinsic mitochondrial pathways, indicating that different mechanisms for regulating intrinsic apoptotic pathways existed in various tissues. Thus, our data highlights the complexity and inconsistency of the HFD-induced apoptotic effects in different tissues. Furthermore, we speculate that the different mRNA expressions of those apoptosis related genes might be attributable to the HFD-induced changes of fat metabolism in various tissues of $P$. fulvidraco.

\section{CONCLUSION}

In summary, we characterized the full-length cDNA sequences of five genes involved in the intrinsic mitochondrial apoptotic pathway (Cycs, Apaf1, Casp9, Casp3a, and Casp3b) and explored their tissue expression profiles in $P$. fulvidraco, which will facilitate further exploration into their functions at the molecular level. HFD-induced expression studies disclose the critical involvement of these genes against nutrient changes, indicating that the processes of apoptosis in various tissues may be modified by an HFD.

\section{DATA AVAILABILITY}

All datasets generated for this study are included in the manuscript and/or the Supplementary Files.

\section{ETHICS STATEMENT}

We assured that the experiments performed on animals followed the ethical guidelines of Huazhong Agricultural University and confirmed that all experimental protocols were approved by Huazhong Agriculture University.

\section{AUTHOR CONTRIBUTIONS}

ZL and D-DL designed the experiment. D-DL conducted the experiment and sample analysis, with the help of S-CL and KW. D-DL and ZL analyzed the data. D-DL drafted the manuscript. ZL revised the manuscript. All the authors approved the manuscript.

\section{FUNDING}

This work was supported by the National Key R\&D Program of China (Grant No. 2018YFD0900400) and by the Fundamental Research Funds for the Central Universities, China (Grant No. 2662018PY089).

\section{ACKNOWLEDGMENTS}

The authors thank the staff of the Aquatic Animal Nutrition and Feed Laboratory of Huazhong Agricultural University for their assistance with excellent sample analysis.

\section{SUPPLEMENTARY MATERIAL}

The Supplementary Material for this article can be found online at: https://www.frontiersin.org/articles/10.3389/fphys. 2019.00921/full\#supplementary-material 


\section{REFERENCES}

Camargo, A., Rangel-Zuniga, O. A., Alcala-Diaz, J., Gomez-Delgado, F., Delgado-Lista, J., García-Carpintero, S., et al. (2017). Dietary fat may modulate adipose tissue homeostasis through the processes of autophagy and apoptosis. Eur. J. Nutr. 56, 1621-1628. doi: 10.1007/s00394-0161208-y

Chiang, L. W., Grenier, J. M., Ettwiller, L., Jenkins, L. P., Ficenec, D., Martin, J., et al. (2001). An orchestrated gene expression component of neuronal programmed cell death revealed by cDNA array analysis. Proc. Natl. Acad. Sci. U.S.A. 98, 2814-2819. doi: 10.1073/pnas.051630598

Espe, M., Holen, E., He, J., Provan, F., Chen, L., Oysæd, K. B., et al. (2015). Hydrolyzed fish proteins reduced activation of caspase- 3 in $\mathrm{H} 2 \mathrm{O} 2$ induced oxidative stressed liver cells isolated from Atlantic salmon (Salmo salar). Springerplus 4, 1-9.

Gao, D., Zhang, X., Wang, H., Wang, Y., and Min, W. (2013). Molecular cloning, immunohistochemical localization, characterization and expression analysis of caspase- 9 from the purse red common carp (Cyprinus carpio) exposed to cadmium. Aquat. Toxicol. 142, 53-62. doi: 10.1016/j.aquatox.2013. 07.017

Hu, W. Y., and Yao, C. L. (2016). Molecular and immune response characterizations of a novel AIF and cytochrome $\mathrm{c}$ in Litopenaeus vannamei defending against WSSV infection. Fish Shellfish Immunol. 56, 84-95. doi: 10. 1016/j.fsi.2016.06.050

Jiang, X., and Wang, X. (2000). Cytochrome cpromotes caspase-9 activation by inducing nucleotide binding to Apaf-1. J. Biol. Chem. 275, 31199-31203. doi: 10.1074/jbc.c000405200

Jones, D. T., Taylor, W. R., and Thornton, J. M. (1992). The rapid generation of mutation data matrices from protein sequences. Bioinformation 8, 275-282. doi: 10.1093/bioinformatics/8.3.275

Kumaresan, V., Ravichandran, G., Nizam, F., Dhayanithi, N. B., Arasu, M. V., Al-Dhabi, N. A., et al. (2016). Multifunctional murrel caspase 1, 2, 3, 8 and 9: conservation, uniqueness and their pathogen-induced expression pattern. Fish Shellfish Immunol. 49, 493-504. doi: 10.1016/j.fsi.2016. 01.008

Li, D. D., Luo, Z., Chen, G. H., Song, Y. F., Wei, C. C., and Pan, Y. X. (2017). Identification of apoptosis-related genes $\mathrm{Bcl} 2$ and $\mathrm{Bax}$ from yellow catfish Pelteobagrus fulvidraco and their transcriptional responses to waterborne and dietborne zinc exposure. Gene 633, 1-8. doi: 10.1016/j.gene.2017. 08.029

Li, H. K., Xu, L. P., Dunbar, J. C., and Dhabuwala, C. B. (2003). Role of mitochondrial cytochrome $\mathrm{c}$ in cocaine-induced apoptosis in rat testes. Urology 61, 646-650. doi: 10.1016/s0090-4295(02)02263-X

Li, M., Ding, Y., Mu, Y., Ao, J., and Chen, X. (2011). Molecular cloning and characterization of caspase-3 in large yellow croaker (Pseudosciaena crocea). Fish Shellfish Immunol. 30, 910-916. doi: 10.1016/j.fsi.2011. 01.018

Liu, X., Kim, C. N., Yang, J., Jemmerson, R., and Wang, X. (1996). Induction of apoptotic program in cell-free extracts: requirement for dATP and cytochrome c. Cell 86, 147-157. doi: 10.1016/s0092-8674(00) 80085-9

Livak, K. J., and Schmittgen, T. D. (2001). Analysis of relative gene expression data using realtime quantitative PCR and the 2- $\Delta \Delta$ Ct method. Methods 25, 402-408. doi: 10.1006/meth.2001.1262

Luo, Z., Liu, Y. J., Mai, K. S., Tian, L. X., Liu, D. H., Tan, X. Y., et al. (2005). Effect of dietary lipid level on growth performance, feed utilization and body composition of grouper Epinephelus coioides juveniles fed isonitrogenous diets in floating netcages. Aquacult. Int. 13, 257-269. doi: 10.1007/s10499-0042478-6

Marsden, V. S., and Strasser, A. (2003). Control of apoptosis in the immune system: Bcl-2, BH3-only proteins and more. Annu. Rev. Immunol. 21, 71-105. doi: 10.1146/annurev.immunol.21.120601.141029

Moraes, J. C., Coope, A., Morari, J., Cintra, D. E., Roman, E. A., Pauli, J. R., et al. (2009). high fat diet induces apoptosis of hypothalamic neurons. PLoS One 4:e5045. doi: 10.1371/journal.pone.0005045

Mu, Y., Xiao, X., Zhang, J., Ao, J., and Chen, X. (2010). Molecular cloning and functional characterization of caspase 9 in large yellow croaker (Pseudosciaena crocea). Dev. Comp. Immunol. 34, 300-307. doi: 10.1016/j.dci.2009. 10.009

Naruse, K., Fukamachi, S., Mitani, H., Kondo, M., Matsuoka, T., Kondo, S., et al. (2000). A detailed linkage map of medaka, Oryzias latipes: comparative genomics and genome evolution. Genetics 154, 1773-1784.

Olivo, S. E., and Hilakivi-Clarke, L. (2005). Opposing effects of prepubertal low-and high fat n-3 polyunsaturated fatty acid diets on rat mammary tumorigenesis. Carcinogenesis 26, 1563-1572. doi: 10.1093/carcin/bgi118

Reis, M. I., do Vale, A., Pinto, C., Nascimento, D. S., Costa-Ramos, C., Silva, D. S., et al. (2007a). First molecular cloning and characterisation of caspase-9 gene in fish and its involvement in a gram negative septicaemia. Mol. Immunol. 44, 1754-1764. doi: 10.1016/j.molimm.2006.07.293

Reis, M. I., Nascimento, D. S., do Vale, A., Silva, M. T., and dos Santos, N. M. (2007b). Molecular cloning and characterisation of sea bass (Dicentrarchus labrax L.) caspase-3 gene. Mol. Immunol. 44, 774-783. doi: 10.1016/j.molimm. 2006.04.028

Sishi, B., Loos, B., Ellis, B., Smith, W., and du Toit, E. F. (2011). Diet-induced obesity alters signalling pathways and induces atrophy and apoptosis in skeletal muscle in a prediabetic rat model. Exp. Physiol. 96, 179-193. doi: 10.1113/ expphysiol.2010.054189

Takle, H., McLeod, A., and Andersen, O. (2006). Cloning and characterization of the executioner caspases 3, 6, 7 and Hsp70 in hyperthermic Atlantic salmon (Salmo salar) embryos. Comp. Biochem. Physiol. 144B, 188-198. doi: 10.1016/j. cbpb.2006.02.006

Tamura, K., Peterson, D., Peterson, N., Stecher, G., Nei, M., and Kumar, S. (2011). MEGA5: molecular evolutionary genetics analysis using maximum likelihood, evolutionary distance, and maximum parsimony methods. Mol. Biol. Evol. 28, 2731-2739. doi: 10.1093/molbev/msr121

Tie, H. M., Wu, P., Jiang, W. D., Liu, Y., Kuang, S. Y., Zeng, Y. Y., et al. (2019). Dietary necleotides supplementation affect the physicochemical properties, amino acid and fatty acid constituents, apoptosis and antioxidant mechanisms in grass carp (Ctenopharyngodon idellus) muscle. Aquaculture 502, 312-325. doi: 10.1016/j.aquaculture.2018.12.045

Vandesompele, J., De Preter, K., Pattyn, F., Poppe, B., VanRoy, N., DePaepe, A., et al. (2002). Accurate normalization of real-time quantitative RT-PCR data by geometric averaging of multiple internal control genes. Genome Biol. 3, 1-11.

Walke, D. W., and Morgan, J. I. (2000). A comparison of the expression and properties of Apaf-1 and Apaf-1L. Brain Res. 886, 73-81. doi: 10.1016/s00068993(00)02916-4

Wang, X. (2001). The expanding role of mitochondria in apoptosis. Genes Dev. 15, $2922-2933$.

Wang, Y., Ausman, L. M., Russell, R. M., Greenberg, A. S., and Wang, X. D. (2008) Increased apoptosis in high fat diet-induced nonalcoholic steatohepatitis in rats is associated with c-Jun NH2-terminal kinase activation and elevated proapoptotic Bax. J. Nutr. 138, 1866-1871. doi: 10.1093/jn/138.10.1866

Wei, C. C., Luo, Z., Song, Y. F., Pan, Y. X., Wu, K., and You, W. J. (2017). Identification of autophagy related genes $1 \mathrm{c} 3$ and atg 4 from yellow catfish Pelteobagrus fulvidraco and their transcriptional responses to waterborne and dietborne zinc exposure. Chemosphere 175, 228-238. doi: 10.1016/j. chemosphere.2017.02.042

Yabu, T., Kishi, S., Okazaki, T., and Yamashita, M. (2001). Characterization of zebrafish caspase- 3 and induction of apoptosis through ceramide generation in fish fathead minnow tailbud cells and zebrafish embryo. Biochem. J. 360, 39-47. doi: 10.1042/bj3600039

Yakovlev, A. G., Ota, K., Wang, G., Movsesyan, V., Bao, W. L., Yoshihara, K., et al. (2001). Differential expression of apoptotic protease-activating factor-1 and caspase- 3 genes and susceptibility to apoptosis during brain development and after traumatic brain injury. J. Neurosci. 21, 7439-7446. doi: 10.1523/jneurosci. 21-19-07439.2001

Ye, H., Xu, M., Chen, L., Tan, X., Chen, S., Zou, C., et al. (2019). Effects of dietary plant protein sources influencing hepatic lipid metabolism and hepatocyte apoptosis in hybrid grouper (Epinephelus lanceolatuso $\sigma^{\top} \times$ Epinephelus fuscoguttatuso). Aquaculture 506, 437-444. doi: 10.1016/j.aquaculture.2019. 03.075

Yin, P., Xie, S., Huo, Y., Guo, T., Fang, H., Zhang, Y., et al. (2019). Effects of dietary oxidized fish oil on growth performance, antioxidant defense system, 
apoptosis and mitochondrial function of juvenile largemouth bass (Micropterus salmoides). Aquaculture 500, 347-358. doi: 10.1016/j.aquaculture.2018.09.009

Zhao, Y., Gul, Y., Li, S., and Wang, W. (2011). Cloning, identification and accurate normalization expression analysis of PPAR $\alpha$ gene by GeNorm in Megalobrama amblycephala. Fish Shellfish Immunol. 31, 462-468. doi: 10.1016/j.fsi.2011. 06.024

Zou, H., Henzel, W. J., Liu, X., Lutschg, A., and Wang, X. (1997). Apaf-1, a human protein homologous to C. elegans CED-4, participates in cytochrome c-dependent activation of caspase-3. Cell 90, 405-413. doi: 10.1016/s0092$8674(00) 80501-2$
Conflict of Interest Statement: The authors declare that the research was conducted in the absence of any commercial or financial relationships that could be construed as a potential conflict of interest.

Copyright $\odot 2019$ Li, Ling, Wu and Luo. This is an open-access article distributed under the terms of the Creative Commons Attribution License (CC BY). The use, distribution or reproduction in other forums is permitted, provided the original author(s) and the copyright owner(s) are credited and that the original publication in this journal is cited, in accordance with accepted academic practice. No use, distribution or reproduction is permitted which does not comply with these terms. 